\title{
Coupled Electromagnetic and Thermal Analysis of Electric Machines
}

\author{
Gabriel Mendes ${ }^{1,2 *}$, Angela Ferreira ${ }^{1}$, and Ednei Miotto ${ }^{2}$ \\ ${ }^{1}$ Research Centre in Digitalization and Intelligent Robotics (CeDRI), Instituto Politécnico de \\ Bragança, Portugal \\ ${ }^{2}$ Federal University of Technology - Parana (UTFPR), Toledo, Brazil
}

\begin{abstract}
This paper deals with the design process of electric machines, proposing a design flowchart which couples the electromagnetic and thermal models of the machine, assisted by finite element techniques. The optimization of an electrical machine, in terms of the energy efficiency and cost reduction requirements, benefits from the coupling design of the electromagnetic and thermal models. It allows the maximization of the current density and, consequently, the torque/power density within thermal limits of the active materials. The proposed coupled electromagneticthermal analysis is demonstrated using a single-phase transformer of 1 kVA. Finite element analysis is carried out via ANSYS Workbench, using Maxwell 3D for the electromagnetic design, with resistive and iron losses directly coupled to a steady-state thermal simulation, in order to determine the temperature rise which, in turn, returns to electromagnetic model for material properties update.
\end{abstract}

\section{Introduction}

The actual trend of the design process of electrical machines is oriented to specific requirements of the applications and is no longer based in standard configurations. From this point of view, design of electrical machines became a multidisciplinary process, typically involving electromagnetic, thermal and mechanical modelling in a highly iterative process.

Despite the electromagnetic and thermal designs being strongly interrelated, the thermal design has been neglected especially for small and medium-sized machines [1]. With nowadays requirements of energy efficiency, cost reduction and power density, the thermal model and its interrelation with, mainly, the electromagnetic design, should no longer be neglected.

A multiphysics model of an electric machine is very complex, especially from an analytical point of view. To assist the multiphysics model, numeric analysis based methods are being used on the initial design process and design optimization of new machines [2].

The use of multiphysics simulation in the design process is especially important, allowing validation of alternatives and changes on a virtual prototype, without the cost and

\footnotetext{
* Corresponding author: apf@ipb.pt
} 
time of building a real one, allowing a competitive advantage between manufacturers of electric machines [3].

The electromagnetic model may be accomplished analytically, through reluctance networks [4-6]. Finite element analysis (FEA) is a numerical method that is being used in electromagnetic models [2,7] to explore design improvements, investigate losses, and simulate different working conditions. Regarding thermal modelling, it can be performed using lumped parameters thermal networks (LPNT) [8, 9], which are widely used [10-12] due to its simplicity and reasonable error. However, the calculation of lumped parameters of complex parts is very difficult and complicated to model with enough accuracy to predict, for instance, hot spots [13]. FEA is a common numeric method in thermal models [14-16], allowing the calculation of the temperature distribution in the model with precision depending essentially on the accuracy of the thermal parameters of the materials involved and empirical relations modelling the convection process. Hence, when the cooling system involves complex fluid flow systems, FEA is no better than LPNT. For these cooling systems, the computation fluid dynamics (CFD) may be used successfully [17-19]. However, CFD requires a lot of resources and time and it is usually employed in hybrid approaches with LPNT and FEA, as proposed in [13] for railway traction motors modelling.

The objective of this work is to develop a coupled electromagnetic and thermal analysis of electric machines, using FEA, emphasizing the key points of the coupling approach. The developed methodology is applied to a simple structure of a single-phase transformer, for demonstrations purposes.

This work is structured as follows: section 2 presents the coupled electromagnetic and thermal analyses, section 3 presents the coupled FEA development for the case study and section 4 concludes the work and discusses future work.

\section{Coupled analysis}

Under the actual trend of multiphysics modelling, several works have been dealing with the coupled field approach [20,21]. In this work, the focus is on coupling the electromagnetic and thermal analyses. The coupled approach allows, for instance, evaluating the electromagnetic model according to the temperature rise. Material properties from magnetic and electric circuits vary non linearly with temperature, and for this reason, the thermal model output can be crucial to evaluate the electromagnetic performance of the electric machines, for instance, the risk of demagnetization of permanent magnet materials or the increase of resistive loss due to temperature rise.

The coupled methodology, as proposed in this paper, is intended to be used as part of the design process of an electrical machine, or it can be useful to evaluate the machine performance in operating conditions that differ from the rated ones. For instance, the derating of the machine in ambient temperature or altitude different from the ones specified for the rated quantities may be more precise, if assisted with an electromagnetic and thermal coupled approach.

The proposed analysis algorithm is summarized in Fig. 1. It starts with the requirements and geometry of the machine. It should be noted that in a design process, the main dimensions of the machine usually results from a sizing approach considering the application constraints. Next, the materials are chosen or specified, i.e., their electromagnetic and thermal data are gathered, e.g., $B-H$ curve, specific loss, electric conductivity, thermal conductivity, etc. In addition, the excitation of the machine needs to be specified. It can be either voltage source, current source or an external circuit with load and power electronics devices. The consideration of a simple magnetostatic model allows the evaluation of some features, such as iron saturation, and guiding to some model 
changes, as for instance, geometries, ferromagnetic material requirements or excitation sources.

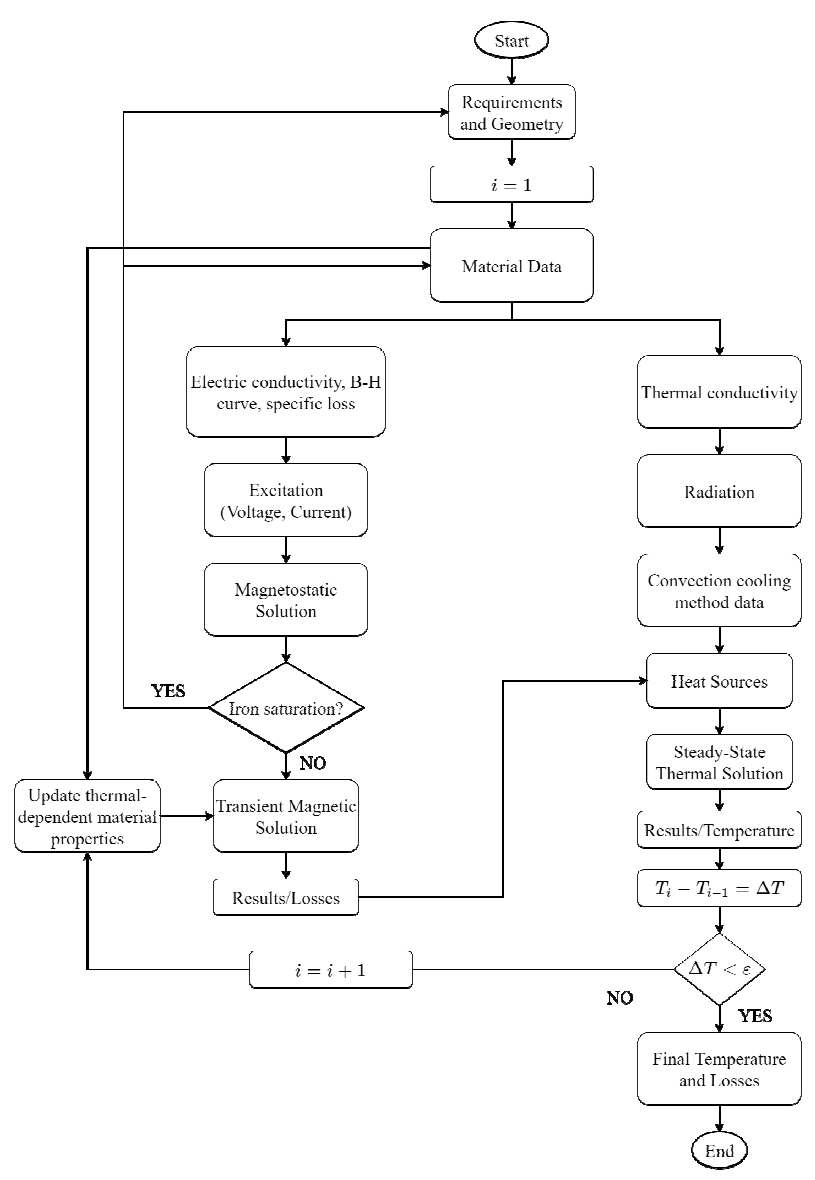

Fig. 1. Proposed electromagnetic and thermal coupled methodology flowchart.

After the magnetostatic solution, a transient solution is performed to evaluate the losses of the machine, iron and resistive losses. For a static machine, these losses will be the heat sources of the thermal model.

The thermal model should incorporate the three modes of heat transfer, conduction, convection and radiation.

According to the Fourier law (1), the heat flow rate due to conduction, $\boldsymbol{q}_{c o n}$, is given by the thermal conductivity $k$ of the material, the surface area $A$ involved and the temperature gradient $\nabla T$.

$$
\boldsymbol{q}_{c o n}=-k A \nabla T
$$

Convection is the heat transfer due to fluid motion. It can be accomplished through natural convection, due to the density difference in the fluid, or can be forced, by external devices, as fans. Heat transfer by convection is proportional to the temperature difference between the surface and the fluid, being expressed by the Newton's law of cooling, given by

$$
\boldsymbol{q}_{\text {conv }}=h A_{s}\left(T_{s}-T_{\infty}\right)
$$


where $h$ is the convection heat transfer coefficient, highly non linear and variable with the physical properties of the fluid (its velocity and also of the surface area, $A_{s}$, in contact with the fluid), $T_{s}$ is the surface temperature, and $T_{\infty}$ the fluid temperature.

Radiation is the energy emitted in the form of electromagnetic waves [22], therefore the thermal radiation of a surface occurs due to its temperature. The heat flux rate for radiation, $\boldsymbol{q}_{\text {rad }}$, is derived from the Stefan-Boltzmann law, which determines the maximum rate of radiation emitted by a surface:

$$
\boldsymbol{q}_{\text {rad }}=\varepsilon \sigma A_{s}\left(T_{s}^{4}-T_{s u r}^{4}\right)
$$

being $\varepsilon$ the surface emissivity, $\sigma$ the Stefan-Boltzmann constant, and $T_{\text {sur }}$ the surrounding temperature. If the machine has only natural convection, the heat transfer by radiation is of considerable importance [23].

The heat generated from the losses is now used to compute the temperature of the machine, which in turn, is used to update the temperature dependent material properties, from which results an update of the losses. The process runs iteratively till the temperature variation, $\Delta T$, from two consecutive iterations is lower than a predefined error, $\varepsilon$.

\section{Electromagnetic and thermal finite element analysis}

Finite element analysis (FEA) is a numerical method to solve complex engineering problems expressed in differential equations, which consists in dividing the analysis domain into finite elements and solve the governing equations for each element, resulting in the solution of the entire domain.

The objective of the subsections 3.1 and 3.2 is to introduce the principles to implement FEA to the problem under study, oriented to available commercial packages (e.g. ANSYS, COMSOL, QuickField). This work exploits Ansys software suite, the Mechanical package [24] is used to implement the thermal simulation and Maxwell package [25] to perform the electromagnetic analysis, while the coupling of the two analyses is accomplished through Ansys Workbench [26].

\subsection{Electromagnetic FEA}

To set up the electromagnetic FEA some topics need to be highlighted: solver type, material data, excitations, boundary conditions, and core losses.

Electromagnetic FEA is established using the differential form of Maxwell's equations. To increase solution performance, available packages offering FEA software have different solver types e.g., magnetostatic, eddy current, transient magnetic, adapted to application modes characterized by different formulations of Maxwell's equations, varying for instance with features to explore and/or time variations of sources. For example, the goal of the electromagnetic model in this work is to obtain the resistive and core losses. Therefore, it requires a solver capable to compute these losses, which in ANSYS Maxwell is available through the Magnetic Transient solver.

Material data of the components are important to enhance the constitutive relations with accuracy, which may be complicated especially when the materials are nonlinear, anisotropic and inhomogeneous, like the iron core of electrical machines. The minimum data required for soft ferromagnetic materials are the $B-H$ and specific loss curves, while for materials used in electrical circuits, the electric conductivity is mandatory and, in a coupled analysis, the material interaction with the coupled variable, e.g. the electric conductivity dependence on the temperature. 
To solve the differential equations, boundary conditions need to be defined, and they are related to the solver type.

The accurate loss calculation is of significant importance in the approach proposed in this work. While the resistive loss calculation is typically enough considering a DC resistance in Joule law, the iron loss is much more complex to calculate due to physical phenomena involved, hysteresis and eddy currents, and also harmonic components in the magnetic flux density. FEA software used in this work has functions implemented able to calculate them, but they can be imprecise, due to uncertainty of the parameters involved, generally empirical. In this regard, the obtained losses should be the object of sensitivity analysis or experimental validation, in order to corroborate the FEA estimation of losses.

\subsection{Thermal FEA}

Similar to the electromagnetic model, the establishment of the thermal model requires the specification of material data involved in the physical mechanisms of heat transfer in the electrical machine, as thermal conductivity, emissivity and convection heat transfer coefficients.

The convection heat transfer coefficient is rather complex to define because it is dependent on many factors, such as the nature of the fluid motion (natural or forced), the fluid itself e.g., water, oil, its velocity and also of the dimensions of the surfaces under consideration. Therefore, the deterministic value of this coefficient is only possible for simple geometries.

The heat sources should be carefully defined, in order to allow an accurate distribution of the heat in the machine and also the identification of possible hot spots.

A steady-state thermal FEA approach allows acquiring the temperature distribution in the machine. With the temperature results, it is possible to check if the insulation material is suitable for the application and operating conditions, in order to prevent shortening the lifetime of the insulation system of the machine.

\subsection{Single-phase transformer analysis}

In order to exploit the coupled electromagnetic and thermal approach as proposed previously, the algorithm was applied to a simple single-phase transformer of $1 \mathrm{kVA}$, $220 \mathrm{~V} / 110 \mathrm{~V}, 50 \mathrm{~Hz}$, with the configuration shown in Fig. 2.

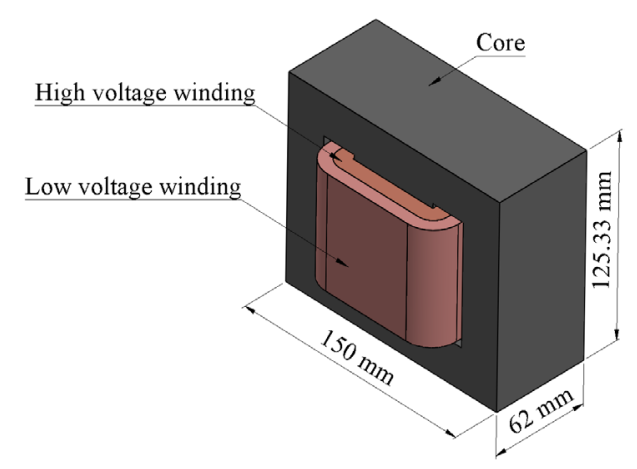

Fig. 2. Single-phase transformer geometry.

After the settlement of the geometry and main characteristics of the materials involved, a 3D electromagnetic analysis was performed, followed by the steady-state thermal, and finally, the coupling interaction was added. 
The material data of the core iron used are shown in Fig. 3, and the electric conductivity of the copper winding, is given by:

$$
\sigma(T)=\sigma_{20} \frac{1}{1+\alpha(T-20)}
$$

where $\sigma_{20}$ is the conductivity of the copper at $20^{\circ} \mathrm{C}$, considered as $58 \times 10^{6} \mathrm{~S} / \mathrm{m}$ and $\alpha$ is the thermal resistivity coefficient, equal to $3.93 \times 10^{-3} /{ }^{\circ} \mathrm{C}$.

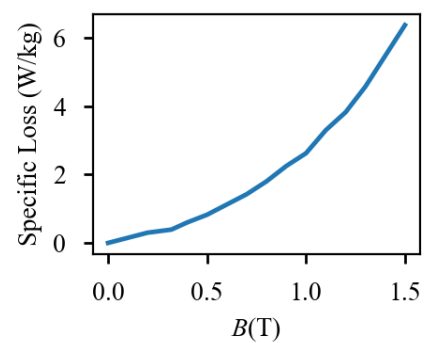

(a)

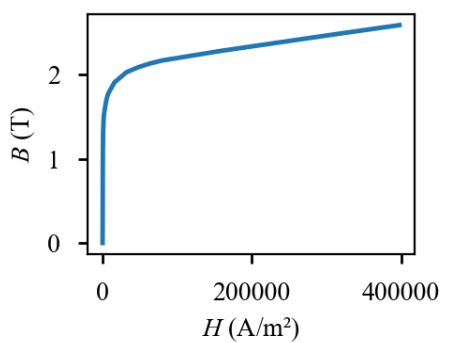

(b)

Fig. 3. Transformer core: specific loss (a), modified from [27] and B-H curve (b) [25].

The resistive losses can be obtained through a magnetostatic solution, however, to calculate the iron losses it is necessary to run a transient solution, therefore the Magnetic Transient solver was used. The excitation of the transformer was implemented by an external circuit, assuming that the transformer is supplied by a $220 \mathrm{~V}$ rms sinusoidal source connected to the high voltage winding. The low voltage winding is connected to a resistive load which imposes the rated condition to the machine.

The model requires insulating boundary conditions from the core and windings to the exterior, by setting the vector potential to zero.

Iron loss is calculated through Bertotti model [28], given by

$$
P_{\text {core }}=K_{h} f\left(B_{\max }\right)^{2}+K_{c}\left(f B_{\max }\right)^{2}+K_{e}\left(f B_{\max }\right)^{1.5}
$$

where $K_{h}, K_{c}$ and $K_{e}$ are the hysteresis, eddy and anomalous loss coefficients, respectively, $f$ is the frequency, and $B_{\max }$ the maximum amplitude of the magnetic flux density. This model is implemented in ANSYS Maxwell, and the above coefficients are calculated with the input data from the specific loss curve, sheet thickness and conductivity.

Since the main goal of this work is the implementation of the coupled analysis, and given the simplicity of the machine, only the resistive loss is considered temperature dependent through DC resistance variation with temperature, although the iron loss may reduce about $20 \%$ with increasing temperature [29].

Fig. 4 shows the iron loss of the transformer in a period of $20 \mathrm{~ms}$, from which it is possible to find the average loss of about $13.76 \mathrm{~W}$. 


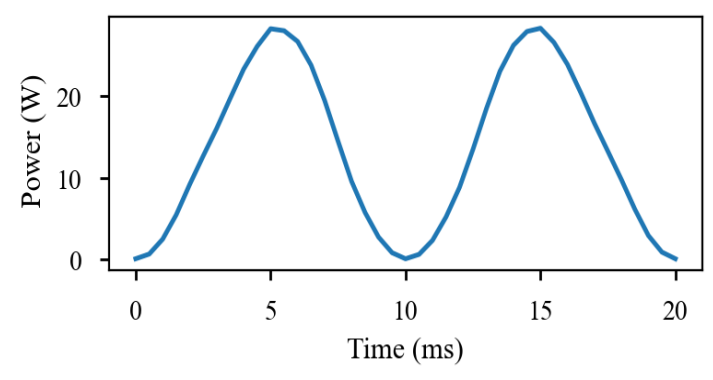

Fig. 4. Transformer iron loss.

The material data used for the thermal model are gathered from [23], being the thermal conductivity of laminated steel core $31 \mathrm{~W} / \mathrm{K} \mathrm{m}$ in the direction of lamination and $0.6 \mathrm{~W} / \mathrm{K} \mathrm{m}$ normal to lamination, and conductivity of copper material is $7 \mathrm{~W} / \mathrm{K} \mathrm{m}$. The emissivity of the core surfaces is considered 0.4 , and the winding is wrapped in black electrical tape with an emissivity of 0.9 .

Convection was set to the core and windings surfaces that are in contact with the air. Because this transformer does not have forced convection, convection heat transfer coefficient is obtained from ANSYS Mechanical database, for natural convection, being temperature-dependent.

The losses obtained from the electromagnetic simulation are imported as internal heat generation and applied into the geometry. Since the losses are obtained from a transient solution, and the thermal solution is performed in a steady-state solver, the losses average are used.

Fig. 5 shows the evolution of the losses during the iteration process. The first three iterations have the most significant variation, especially the second one, where the average temperature raised $14.08 \%$, and the total loss $22.44 \%$.

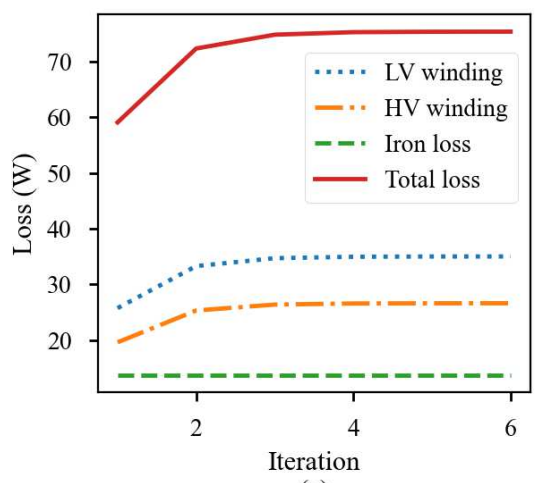

(a)

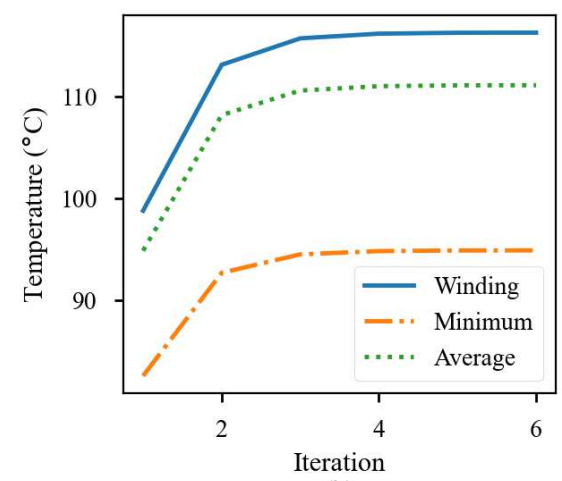

(b)

Fig. 5. Transformer results: (a) losses and (b) temperature.

In order to validate the obtained losses, iron and resistive losses from the coupled thermal and electromagnetic analysis are compared with the losses determined experimentally through no-load and short-circuit tests. The later was performed at the ambient temperature, in order to validate the initial value of the resistive losses (at ambient temperature), being the final value estimated by measuring the winding resistances at the final temperature (i.e., after the transformer reach the steady-state temperature, estimated after two hours operating at rated condition). It should be noted that the temperature 
dependence of the iron loss with the temperature is neglected in this work, as previously stated. The obtained values are presented in Table 1.

Table 1. FEA and experimental transformer losses.

\begin{tabular}{|l|c|c|c|}
\hline & $\begin{array}{c}\text { Resistive loss } \\
\left(\mathbf{2 0}^{\circ} \mathbf{C}\right)(\mathbf{W})\end{array}$ & $\begin{array}{c}\text { Resistive loss } \\
\left(\mathbf{1 1 6}^{\mathbf{}} \mathbf{C}\right)(\mathbf{W})\end{array}$ & $\begin{array}{c}\text { Iron loss } \\
(\mathbf{W})\end{array}$ \\
\hline FEA & 45.36 & 61.64 & 13.76 \\
\hline Experimental & 41 & 56.46 & 13 \\
\hline Error $(\%)$ & 10.63 & 9.17 & 5.85 \\
\hline
\end{tabular}

From the errors obtained, and given some uncertainty in the data of the active materials and dimensions of the physical transformer (number of turns, and iron specifications, e.g.), the modelling performed is acceptable.

Regarding the coupling analysis, it allowed the estimation of the resistive loss with higher precision than the ones that could be obtained without coupling the models. Without the coupled analysis the winding steady-state temperature would be around $15^{\circ} \mathrm{C}$ lower than the actual temperature. For machines with more heat sources and complex geometries, this error can be larger and decisive in their performance.

The simplicity of the single-phase transformer has facilitated the understanding and the establishment of the key concepts of the electromagnetic and thermal coupling process, and emphasized its importance in the design of electric machines.

\section{Conclusions and future work}

This work developed a simple but effective approach for coupling the electromagnetic and thermal models of electric machines. The objective of this method is to be able to help the design process, calculating the heat sources, the temperature distribution in the machine and their interaction. By this way, this approach can assist design choices and improvements not requiring building a prototype, saving time and resources. Additionally, it can assist the derating process of the machine, by simulating different working conditions as different ambient temperature, air pressure and applications.

The method developed was applied to a study case of a single-phase transformer, demonstrating the interaction between the electromagnetic and thermal models. With the results is possible to identify improvements that can be done in the real model, improving its performance. To fully validate the proposed method, experimental measurements of the transformer temperature are required. Therefore, future work should validate experimentally this method, using temperature sensors and thermography. It is also suggested to investigate the temperature dependence of the iron loss.

\section{Acknowledgment}

This work has been supported by FCT - Fundação para a Ciência e Tecnologia within the Project Scope: UIDB/05757/2020.

\section{References}

1. D. Staton, A. Boglietti, A. Cavagnino, Solving the more difficult aspects of electric motor thermal analysis (IEMDC 2003 - IEEE Int. Electr. Mach. Drives Conf. 2, 747755, 2003)

2. E. Schmidt, Int. J. Comput. Math. Electr. Electron. Eng. 30, 1899-1913 (2011) 
3. R. Marius, P. Zhou, D. Lin, D. Ionel, M. Popescu, F. Blaabjerg, V. Rallabandi, D. Staton, Multiphysics Simulation by Design for Electrical Machines, Power Electronics, and Drives (John Wiley \& Sons, 2018)

4. T. Ohinata, K. Arimatsu, IEEE Trans. Magn. 55, 1-6 (2019)

5. E. M. Barhoumi, F. Wurtz, C. Chillet, B. Ben Salah, Reluctance network model for linear switched reluctance motor (12th Int. Multi-Conference Syst. Signals Devices, SSD 2015, 1-4, 2015).

6. A. A. Diriye, Y. Amara, G. Barakat, Three-Dimensional Modeling of Permanents Magnets Synchronous Machines Using a 3D Reluctance Network (Proc. - 2018 23rd Int. Conf. Electr. Mach. ICEM 2018, 2304-2310, 2018)

7. C. Freitag, Magnetic Properties of Electrical Steel, Power Transformer Core Losses and Core Design Concepts (Elektrotechnik und Informationstechnik des Karlsruher Institutes für Technologie KIT, 2017)

8. M. Popescu, D. A. Staton, A. Boglietti, A. Cavagnino, D. Hawkins, J. Goss, IEEE Trans. Ind. Appl. 52, 2167-2175 (2016)

9. A. Boglietti, A. Cavagnino, D. Staton, M. Shanel, M. Mueller, C. Mejuto, IEEE Trans. Ind. Electron. 56, 871-882 (2009)

10. T. A. Jankowski, F. C. Prenger, D. D. Hill, S. R. O'Bryan, K. K. Sheth, E. B. Brookbank, D. F. A. Hunt, Y. A. Orrego, IEEE Trans. Ind. Electron. 57, 4043-4054 (2010)

11. A. Boglietti, A. Cavagnino, M. Lazzari, M. Pastorelli, IEEE Trans. Ind. Appl. 39, 945952 (2003)

12. M. Cavazzuti, G. Gaspari, S. Pasquale, E. Stalio, Appl. Therm. Eng. 157, 113733 (2019)

13. S. Nategh, H. Zhang, O. Wallmark, A. Boglietti, T. Nassen, M. Bazant, IEEE Trans. Ind. Electron. 66, 79-89 (2019)

14. Y. Xie, Y. Wang, Appl. Therm. Eng. 66, 25-34 (2014)

15. M. Allahbakhshi, M. Akbari, Appl. Therm. Eng. 100, 714-720 (2016)

16. D. Sarkar, A. K. Naskar, Int. J. Electr. Power Energy Syst. 44, 938-948 (2013)

17. E. Galloni, P. Parisi, F. Marignetti, G. Volpe, Therm. Sci. Eng. Prog. 8, 470-476 (2018)

18. M. Satrústegui, G. Artetxe, I. Elosegui, M. Martinez-Iturralde, J. C. Ramos, Appl. Therm. Eng. 129, 93-105 (2018)

19. J. Pyrhönen, P. Lindh, M. Polikarpova, E. Kurvinen, V. Naumanen, Appl. Therm. Eng. 76, 245-251 (2015)

20. S. Mezani, N. Takorabet, B. Laporte, IEEE Trans. Magn. 41, 1572-1575 (2005)

21. A. Fasquelle, J. Le Besnerais, S. Harmand, M. Hecquet, S. Brisset, P. Brochet, A. Randria, Appl. Therm. Eng. 30, 2788-2795 (2010)

22. Y. A. Cengel, Heat Transfer A Practical Approach, 2nd ed. (Mcgraw-Hill, 2002).

23. J. Pyrhönen, T. Jokinen, V. Hrabovcová, Design of Rotating Electrical Machines, (John Wiley \& Sons, 2008)

24. Ansys, Ansys ${ }^{\circledR}$ Academic Research Mechanical, Release 2019 R3, (2019).

25. Ansys, Ansys ${ }^{\circledR}$ Academic Research Maxwell, Release 2019 R3, (2019).

26. Ansys, Ansys ${ }^{\circledR}$ Academic Research Workbench, Release 2019 R3, (2019).

27. Ugine Aciers de Chatillon et Gueugnon, Fe V 470 - 50 HA, (2011). 
28. G. Bertotti, IEEE Trans. Magn. 24, 621-630 (1988)

29. J. Driesen, R. Belmans, K. Hameyer, Methodologies for coupled transient electromagnetic-thermal finite element modeling of electrical energy transducers (IEMDC 2001 - IEEE Int. Electr. Mach. Drives Conf. ,681-686, 2001) 